\title{
Hermite-Hadamard type and Fejér type inequalities for general weights (I)
}

Shiow-Ru Hwang ${ }^{1}$, Kuei-Lin Tseng ${ }^{2 *}$ and Kai-Chen Hsu²

"Correspondence:

kltseng@mail.au.edu.tw;

kltseng1@gmail.com

${ }^{2}$ Department of Applied

Mathematics, Aletheia University, Tamsui, New Taipei, 25103, Taiwan

Full list of author information is

available at the end of the article

\section{Abstract}

In this paper, we establish some weighted versions of the Hermite-Hadamard type and Fejér type inequalities and from which generalize Hermite-Hadamard inequality, Fejér inequality and several results in (Dragomir in J. Math. Anal. Appl. 167:49-56, 1992; Yang and Hong in Tamkang. J. Math. 28(1):33-37, 1997; Yang and Tseng in J. Math. Anal. Appl. 239:180-187, 1999; Yang and Tseng in Taiwan. J. Math. 7(3):433-440, 2003).

MSC: Primary 26D15; secondary 26A51

Keywords: Hermite-Hadamard inequality; Fejér inequality; convex function

\section{Introduction}

Throughout this paper, let $a<b$ in $\mathbb{R}, c<d$ in $\mathbb{R}, f:[a, b] \rightarrow \mathbb{R}$ be convex, the weight function $p:[a, b] \rightarrow[0, \infty)$ be integrable and symmetric about the line $s=\frac{a+b}{2}$, the weight function $p_{1}:[c, d] \rightarrow[0, \infty)$ be integrable and symmetric about the line $s=\frac{c+d}{2}$ and let the weight function $g:[c, d] \rightarrow[a, b]$ be continuous and symmetric about the point $\left(\frac{c+d}{2}, g\left(\frac{c+d}{2}\right)\right)$, that is, $\frac{1}{2}[g(s)+g(c+d-s)]=g\left(\frac{c+d}{2}\right)(s \in[c, d])$. Define the following functions on $[0,1]$ :

$$
\begin{aligned}
& H(t)=\frac{1}{b-a} \int_{a}^{b} f\left(t s+(1-t) \frac{a+b}{2}\right) d s ; \\
& H_{g}(t)=\frac{1}{d-c} \int_{c}^{d} f\left(\operatorname{tg}(s)+(1-t) g\left(\frac{c+d}{2}\right)\right) d s ; \\
& W H(t)=\int_{a}^{b} f\left(t s+(1-t) \frac{a+b}{2}\right) p(s) d s ; \\
& W H_{g}(t)=\int_{c}^{d} f\left(\operatorname{tg}(s)+(1-t) g\left(\frac{c+d}{2}\right)\right) p_{1}(s) d s ; \\
& F(t)=\frac{1}{(b-a)^{2}} \int_{a}^{b} \int_{a}^{b} f(t s+(1-t) u) d s d u ; \\
& F_{g}(t)=\frac{1}{(d-c)^{2}} \int_{c}^{d} \int_{c}^{d} f(\operatorname{tg}(s)+(1-t) g(u)) d s d u ; \\
& W F(t)=\int_{a}^{b} \int_{a}^{b} f(t s+(1-t) u) p(s) p(u) d s d u ; \\
& W F_{g}(t)=\int_{c}^{d} \int_{c}^{d} f(\operatorname{tg}(s)+(1-t) g(u)) p_{1}(s) p_{1}(u) d s d u ;
\end{aligned}
$$




$$
\begin{aligned}
P(t)= & \frac{1}{2(b-a)} \int_{a}^{b}\left[f\left(\left(\frac{1+t}{2}\right) a+\left(\frac{1-t}{2}\right) s\right)\right. \\
& \left.+f\left(\left(\frac{1+t}{2}\right) b+\left(\frac{1-t}{2}\right) s\right)\right] d s ; \\
P_{g}(t)= & \frac{1}{2(d-c)} \int_{c}^{d}\left[f\left((1-t) g\left(\frac{s+c}{2}\right)+\operatorname{tg}(c)\right)\right. \\
& \left.+f\left((1-t) g\left(\frac{s+d}{2}\right)+\operatorname{tg}(d)\right)\right] d s ; \\
W P(t)= & \frac{1}{2} \int_{a}^{b}\left[f\left(\left(\frac{1+t}{2}\right) a+\left(\frac{1-t}{2}\right) s\right) p\left(\frac{s+a}{2}\right)\right. \\
& \left.+f\left(\left(\frac{1+t}{2}\right) b+\left(\frac{1-t}{2}\right) s\right) p\left(\frac{s+b}{2}\right)\right] d s
\end{aligned}
$$

and

$$
\begin{aligned}
W P_{g}(t)= & \frac{1}{2} \int_{c}^{d}\left[f\left((1-t) g\left(\frac{s+c}{2}\right)+\operatorname{tg}(c)\right) p_{1}\left(\frac{s+c}{2}\right)\right. \\
& \left.+f\left((1-t) g\left(\frac{s+d}{2}\right)+\operatorname{tg}(d)\right) p_{1}\left(\frac{s+d}{2}\right)\right] d s .
\end{aligned}
$$

\section{Remark 1}

(1) Let $c=a, d=b$ and the function $g(s)=s$ on $[a, b]$. Then the functions $H_{g}(t)=H(t)$, $F_{g}(t)=F(t)$ and $P_{g}(t)=P(t)$ on $[0,1]$.

(2) Let $c=a, d=b$ and let the functions $g(s)=s$ and $p_{1}(s)=p(s)$ on $[a, b]$. Then the functions $W H_{g}(t)=W H(t), W F_{g}(t)=W F(t)$ and $W P_{g}(t)=W P(t)$ on $[0,1]$.

In 1893, Hadamard [1] established the following inequality.

If the function $f$ is defined as above, then

$$
f\left(\frac{a+b}{2}\right) \leq \frac{1}{b-a} \int_{a}^{b} f(s) d s \leq \frac{f(a)+f(b)}{2}
$$

is known as Hermite-Hadamard inequality.

See [2-8] and [9-16] for some results in which this famous integral inequality (1.1) is generalized, improved and extended.

Dragomir [2] established the following Hermite-Hadamard type inequalities related to the functions $H, F$, which refine the first inequality of (1.1).

Theorem A Let the functions $f, H$ be defined as in the first page. Then the function $H$ is convex, increasing on $[0,1]$, and for all $t \in[0,1]$, we have

$$
f\left(\frac{a+b}{2}\right)=H(0) \leq H(t) \leq H(1)=\frac{1}{b-a} \int_{a}^{b} f(s) d s .
$$

Theorem B Let the functions $f, F$ be defined as in the first page. Then:

(1) The function $F$ is convex on $[0,1]$, symmetric about $\frac{1}{2}, F$ is decreasing on $\left[0, \frac{1}{2}\right]$ and 
increasing on $\left[\frac{1}{2}, 1\right]$, and we have

$$
\sup _{t \in[0,1]} F(t)=F(0)=F(1)=\frac{1}{b-a} \int_{a}^{b} f(s) d s
$$

and

$$
\inf _{t \in[0,1]} F(t)=F\left(\frac{1}{2}\right)=\frac{1}{(b-a)^{2}} \int_{a}^{b} \int_{a}^{b} f\left(\frac{s+u}{2}\right) d s d u
$$

(2) We have

$$
f\left(\frac{a+b}{2}\right) \leq F\left(\frac{1}{2}\right) ; \quad H(t) \leq F(t), \quad t \in[0,1]
$$

Yang and Hong [12] established the following Hermite-Hadamard type inequality related to the function $P$, which refines the second inequality of (1.1).

Theorem $\mathbf{C}$ Let the functions $f, P$ be defined as in the first and second pages. Then the function $P$ is convex, increasing on $[0,1]$, and for all $t \in[0,1]$, we have

$$
\frac{1}{b-a} \int_{a}^{b} f(s) d s=P(0) \leq P(t) \leq P(1)=\frac{f(a)+f(b)}{2} .
$$

In 1906, Fejér [8] established the following weighted generalization of Hermite-Hadamard inequality (1.1).

Theorem D Let the functions $f, p$ be defined as in the first page. Then

$$
f\left(\frac{a+b}{2}\right) \int_{a}^{b} p(s) d s \leq \int_{a}^{b} f(s) p(s) d s \leq \frac{f(a)+f(b)}{2} \int_{a}^{b} p(s) d s
$$

is known as the Fejér inequality.

Yang and Tseng $[13,16]$ established the following Fejér type inequalities related to the functions $W H, W P, W F$ and which generalize Theorems A-C and refine Fejér inequality (1.5).

Theorem E [13] Let the functions $f, p, W H, W P$ be defined as in the first and second pages. Then the functions $\mathrm{Hg}, \mathrm{Pg}$ are convex and increasing on $[0,1]$, and for all $t \in[0,1]$, we have

$$
\begin{aligned}
f\left(\frac{a+b}{2}\right) \int_{a}^{b} g(s) d s & =W H(0) \leq W H(t) \leq W H(1) \\
& =\int_{a}^{b} f(s) p(s) d s \\
& =W P(0) \leq W P(t) \leq W P(1) \\
& =\frac{f(a)+f(b)}{2} \int_{a}^{b} p(s) d s .
\end{aligned}
$$


Theorem F [16] Let the functions $f, p, W H$, WF be defined as in the first and second pages. Then we have the following results:

(1) The function WF is convex on $[0,1]$ and symmetric about $\frac{1}{2}$.

(2) The function WF is decreasing on $\left[0, \frac{1}{2}\right]$ and increasing on $\left[\frac{1}{2}, 1\right]$,

$$
\sup _{t \in[0,1]} W F(t)=W F(0)=W F(1)=\int_{a}^{b} f(s) p(s) d s
$$

and

$$
\inf _{t \in[0,1]} W F(t)=W F\left(\frac{1}{2}\right)=\int_{a}^{b} \int_{a}^{b} f\left(\frac{s+u}{2}\right) p(s) p(u) d s d u .
$$

(3) We have:

$$
f\left(\frac{a+b}{2}\right)\left(\int_{a}^{b} p(s) d s\right)^{2} \leq W F\left(\frac{1}{2}\right)
$$

and

$$
W H(t) \int_{a}^{b} p(s) d s \leq W F(t)
$$

for all $t \in[0,1]$.

In this paper, we establish some weighted versions of the Hermite-Hadamard type and Fejér type inequalities related to the functions $H_{g}, F_{g}, P_{g}, W H_{g}, W F_{g}, W P_{g}$, which generalize the inequality (1.1) and Theorems A-F.

\section{Hermite-Hadamard type inequalities for general weights}

In this section, we establish some Hermite-Hadamard type inequalities for general weights, which generalize the Hermite-Hadamard inequality (1.1) and Theorems A-C.

In order to prove the results in this paper, we need the following lemmas.

Lemma 1 (see [9]) Let the function $f$ be defined as in the first page and let $a \leq A \leq C \leq$ $D \leq B \leq b$ with $A+B=C+D$. Then

$$
f(C)+f(D) \leq f(A)+f(B) .
$$

The assumptions in Lemma 1 can be weakened as in the following lemma.

Lemma 2 Let the function $f$ be defined as in the first page and let $A, B, C, D \in[a, b]$ with $A+B=C+D$ and $|C-D| \leq|A-B|$. Then

$$
f(C)+f(D) \leq f(A)+f(B)
$$

Proof Without loss of generalization, we can assume that $a \leq A \leq B \leq b$ and $a \leq C \leq$ $D \leq b$. For $|C-D| \leq|A-B|$, we have $A-B \leq C-D$ and $D-C \leq B-A$. Hence, by the 
above inequalities and $A+B=C+D$, we get $a \leq A \leq C \leq D \leq B \leq b$. Thus, the proof is completed by Lemma 1 .

Now, we are ready to state and prove our new results.

Theorem 1 Let the functions $f, g$ be defined as in the first page. Then:

(1) We have

$$
f\left(g\left(\frac{c+d}{2}\right)\right) \leq \frac{1}{d-c} \int_{c}^{d} f(g(s)) d s
$$

(2) As the function $g$ is monotonic on $[c, d]$, we obtain

$$
\frac{1}{d-c} \int_{c}^{d} f(g(s)) d s \leq \frac{f(g(c))+f(g(d))}{2} .
$$

Proof

(1) Using simple techniques of integration, we have the identity

$$
\frac{1}{d-c} \int_{c}^{d} f(g(s)) d s=\frac{1}{d-c} \int_{c}^{\frac{c+d}{2}}[f(g(s))+g(c+d-s)] d s .
$$

Next, using $g(s)+g(c+d-s)=2 g\left(\frac{c+d}{2}\right)$ and

$$
\left|g\left(\frac{c+d}{2}\right)-g\left(\frac{c+d}{2}\right)\right| \leq|g(s)-g(c+d-s)|
$$

in Lemma 2, we obtain

$$
2 f\left(g\left(\frac{c+d}{2}\right)\right) \leq f(g(s))+f(g(c+d-s))
$$

where $s \in[c, d]$. Integrating the above inequality over $s$ on $\left[c, \frac{c+d}{2}\right]$, dividing both sides by $d-c$ and using the above identity, we obtain the inequality (2.1).

(2) For the monotonicity of $g$, we have $|g(s)-g(c+d-s)| \leq|g(c)-g(d)|$ for all $s \in[c, d]$. Using the above inequality and $g(s)+g(c+d-s)=g(c)+g(d)$ in Lemma 2, we obtain

$$
f(g(s))+f(g(c+d-s)) \leq f(g(c))+f(g(d)),
$$

where $s \in[c, d]$. Integrating the above inequality over $s$ on $\left[c, \frac{c+d}{2}\right]$, dividing both sides by $d-c$ and using the inequality (2.3), we obtain the inequality (2.2). This completes the proof.

Remark 2 In Theorem 1 , let $c=a, d=b$ and the function $g(s)=s$ on $[a, b]$. Then Theorem 1 reduces to the Hermite-Hadamard inequality (1.1).

Theorem 2 Let the functions $f, g, H_{g}$ be defined as in the first page. Then:

(1) The function $H_{g}$ is convex on $[0,1]$. 
(2) The function $H_{g}$ is increasing on $[0,1]$ and for all $t \in[0,1]$, we have

$$
f\left(g\left(\frac{c+d}{2}\right)\right)=H_{g}(0) \leq H_{g}(t) \leq H_{g}(1)=\frac{1}{d-c} \int_{c}^{d} f(g(s)) d s .
$$

Proof

(1) It is easily observed from the convexity of $f$ that the function $H_{g}$ is convex on $[0,1]$.

(2) Using simple techniques of integration, we have the following identity:

$$
\begin{aligned}
H_{g}(t)= & \frac{1}{d-c} \int_{c}^{\frac{c+d}{2}}\left[f\left(\operatorname{tg}(s)+(1-t) g\left(\frac{c+d}{2}\right)\right)\right. \\
& \left.+f\left(\operatorname{tg}(c+d-s)+(1-t) g\left(\frac{c+d}{2}\right)\right)\right] d s
\end{aligned}
$$

for all $t \in[0,1]$. Let $t_{1}<t_{2}$ in $[0,1]$. Since $g(s)+g(c+d-s)=2 g\left(\frac{c+d}{2}\right)(s \in[c, d])$, we obtain

$$
\begin{aligned}
& {\left[t_{1} g(s)+\left(1-t_{1}\right) g\left(\frac{c+d}{2}\right)\right]+\left[t_{1} g(c+d-s)+\left(1-t_{1}\right) g\left(\frac{c+d}{2}\right)\right]} \\
& \quad=\left[t_{2} g(s)+\left(1-t_{2}\right) g\left(\frac{c+d}{2}\right)\right]+\left[t_{2} g(c+d-s)+\left(1-t_{2}\right) g\left(\frac{c+d}{2}\right)\right]
\end{aligned}
$$

and

$$
\begin{aligned}
& \left|\left[t_{1} g(s)+\left(1-t_{1}\right) g\left(\frac{c+d}{2}\right)\right]-\left[t_{1} g(c+d-s)+\left(1-t_{1}\right) g\left(\frac{c+d}{2}\right)\right]\right| \\
& \quad=t_{1}|g(s)-g(c+d-s)| \\
& \quad \leq t_{2}|g(s)-g(c+d-s)| \\
& \quad=\left|\left[t_{2} g(s)+\left(1-t_{2}\right) g\left(\frac{c+d}{2}\right)\right]-\left[t_{2} g(c+d-s)+\left(1-t_{2}\right) g\left(\frac{c+d}{2}\right)\right]\right|
\end{aligned}
$$

for all $s \in\left[c, \frac{c+d}{2}\right]$. Therefore, by Lemma 2, the following inequality holds for all $s \in\left[c, \frac{c+d}{2}\right]$ :

$$
\begin{aligned}
& f\left(t_{1} g(s)+\left(1-t_{1}\right) g\left(\frac{c+d}{2}\right)\right)+f\left(t_{1} g(c+d-s)+\left(1-t_{1}\right) g\left(\frac{c+d}{2}\right)\right) \\
& \quad \leq f\left(t_{2} g(s)+\left(1-t_{2}\right) g\left(\frac{c+d}{2}\right)\right)+f\left(t_{2} g(c+d-s)+\left(1-t_{2}\right) g\left(\frac{c+d}{2}\right)\right)
\end{aligned}
$$

where $A=t_{2} g(s)+\left(1-t_{2}\right) g\left(\frac{c+d}{2}\right), B=t_{2} g(c+d-s)+\left(1-t_{2}\right) g\left(\frac{c+d}{2}\right), C=t_{1} g(s)+\left(1-t_{1}\right) g\left(\frac{c+d}{2}\right)$ and $t_{1} g(c+d-s)+\left(1-t_{1}\right) g\left(\frac{c+d}{2}\right)$. Integrating the above inequality over $s$ on $\left[c, \frac{c+d}{2}\right]$, dividing both sides by $d-c$ and using the above identity, we have

$$
H_{g}\left(t_{1}\right) \leq H_{g}\left(t_{2}\right)
$$

Thus, the function $H_{g}$ is increasing on $[0,1]$ and from which the inequality (2.6) holds. This completes the proof. 


\section{Remark 3}

(1) In Theorem 2, the inequality (2.6) refines the inequality (2.1).

(2) In Theorem 2, let $c=a, d=b$ and the function $g(s)=s$ on $[a, b]$. Then the functions $H_{g}(t)=H(t)(t \in[0,1])$ and Theorem 1 reduces to Theorem A.

Theorem 3 Let the functions $f, g, P_{g}$ be defined as in the first and second pages. Then:

(1) The function $P_{g}$ is convex on $[0,1]$.

(2) The function $P_{g}$ is increasing on $[0,1]$ and, for all $t \in[0,1]$, we have

$$
\frac{1}{d-c} \int_{c}^{d} f(g(s)) d s=P_{g}(0) \leq P_{g}(t) \leq P_{g}(1)=\frac{f(g(c))+f(g(d))}{2}
$$

as the function $g$ is monotonic on $[c, d]$.

\section{Proof}

(1) It is easily observed from the convexity of $f$ that the function $P_{g}$ is convex on $[0,1]$.

(2) Using simple techniques of integration, we have the following identity:

$$
\begin{aligned}
P_{g}(t)= & \frac{1}{d-c} \int_{c}^{\frac{c+d}{2}}[f(\operatorname{tg}(c)+(1-t) g(s)) \\
& +f(\operatorname{tg}(d)+(1-t) g(c+d-s))] d s
\end{aligned}
$$

for all $t \in[0,1]$. Let $t_{1}<t_{2}$ in $[0,1]$. Since $g(s)+g(c+d-s)=2 g\left(\frac{c+d}{2}\right)(s \in[c, d])$ and the monotonicity of $g$ on $[c, d]$, we obtain

$$
\begin{aligned}
& |g(s)-g(c+d-s)| \leq|g(c)-g(d)| \\
& {\left[t_{1} g(c)+\left(1-t_{1}\right) g(s)\right]+\left[t_{1} g(d)+\left(1-t_{1}\right) g(c+d-s)\right]} \\
& \quad=\left[t_{2} g(c)+\left(1-t_{2}\right) g(s)\right]+\left[t_{2} g(d)+\left(1-t_{2}\right) g(c+d-s)\right]
\end{aligned}
$$

and

$$
\begin{aligned}
& \left|\left[t_{1} g(c)+\left(1-t_{1}\right) g(s)\right]-\left[t_{1} g(d)+\left(1-t_{1}\right) g(c+d-s)\right]\right| \\
& \quad=\left|t_{1}[g(c)-g(d)]+\left(1-t_{1}\right)[g(s)-g(c+d-s)]\right| \\
& \quad=t_{1}|g(c)-g(d)|+\left(1-t_{1}\right)|g(s)-g(c+d-s)| \\
& \quad \leq t_{1}|g(c)-g(d)|+\left(1-t_{1}\right)|g(s)-g(c+d-s)| \\
& \quad=\left|\left[t_{2} g(c)+\left(1-t_{2}\right) g(s)\right]-\left[t_{2} g(d)+\left(1-t_{2}\right) g(c+d-s)\right]\right|
\end{aligned}
$$

for all $s \in\left[c, \frac{c+d}{2}\right]$. Therefore, by Lemma 2, the following inequality holds for all $s \in\left[c, \frac{c+d}{2}\right]$ :

$$
\begin{aligned}
& f\left(t_{1} g(c)+\left(1-t_{1}\right) g(s)\right)+f\left(t_{1} g(d)+\left(1-t_{1}\right) g(c+d-s)\right) \\
& \quad \leq f\left(t_{2} g(c)+\left(1-t_{2}\right) g(s)\right)+f\left(t_{2} g(d)+\left(1-t_{2}\right) g(c+d-s)\right)
\end{aligned}
$$

where $A=t_{2} g(c)+\left(1-t_{2}\right) g(s), B=t_{2} g(d)+\left(1-t_{2}\right) g(c+d-s), C=t_{1} g(c)+\left(1-t_{1}\right) g(s)$ and $t_{1} g(d)+\left(1-t_{1}\right) g(c+d-s)$. Integrating the above inequality over $s$ on $\left[c, \frac{c+d}{2}\right]$, dividing both 
sides by $d-c$ and using the above identity, we have

$$
P_{g}\left(t_{1}\right) \leq P_{g}\left(t_{2}\right)
$$

Thus, the function $P_{g}$ is increasing on $[0,1]$ and from which the inequality $(2.8)$ holds. This completes the proof.

\section{Remark 4}

(1) In Theorem 3, the inequality (2.8) refines the inequality (2.2).

(2) In Theorem 3, let $c=a, d=b$ and the function $g(s)=s$ on $[a, b]$. Then the functions $P_{g}(t)=P(t)(t \in[0,1])$ and Theorem 3 reduces to Theorem $\mathrm{C}$.

Theorem 4 Let the functions $f, g, H_{g}, F_{g}$ be defined as in the first page. Then we have the following results:

(1) The function $F_{g}$ is convex on $[0,1]$ and symmetric about $\frac{1}{2}$.

(2) The function $F_{g}$ is decreasing on $\left[0, \frac{1}{2}\right]$ and increasing on $\left[\frac{1}{2}, 1\right]$,

$$
\sup _{t \in[0,1]} F_{g}(t)=F_{g}(0)=F_{g}(1)=\frac{1}{d-c} \int_{c}^{d} f(g(s)) d s
$$

and

$$
\begin{aligned}
\inf _{t \in[0,1]} F_{g}(t) & =F_{g}\left(\frac{1}{2}\right) \\
& =\frac{1}{(d-c)^{2}} \int_{c}^{d} \int_{c}^{d} f\left(\frac{g(s)+g(u)}{2}\right) d s d u .
\end{aligned}
$$

(3) We have:

$$
H_{g}(t) \leq F_{g}(t) \quad(t \in[0,1])
$$

and

$$
f\left(g\left(\frac{c+d}{2}\right)\right) \leq F_{g}\left(\frac{1}{2}\right)
$$

\section{Proof}

(1) It is easily observed from the convexity of $f$ that the function $F_{g}$ is convex on $[0,1]$.

By changing variables, we have

$$
F_{g}(t)=F_{g}(1-t), \quad t \in[0,1]
$$

from which we get that the function $F_{g}$ is symmetric about $\frac{1}{2}$.

(2) Let $t_{1}<t_{2}$ in $\left[0, \frac{1}{2}\right]$. Then $t_{2}+\left(1-t_{2}\right)=t_{1}+\left(1-t_{1}\right),\left|t_{2}-\left(1-t_{2}\right)\right| \leq\left|t_{1}-\left(1-t_{1}\right)\right|$ and by Lemma 2, we obtain

$$
\frac{1}{2}\left[F_{g}\left(t_{2}\right)+F_{g}\left(1-t_{2}\right)\right] \leq \frac{1}{2}\left[F_{g}\left(t_{1}\right)+F_{g}\left(1-t_{1}\right)\right] .
$$


Using the symmetry of $F_{g}$, we have

$$
\begin{aligned}
& F_{g}\left(t_{1}\right)=\frac{1}{2}\left[F_{g}\left(t_{1}\right)+F_{g}\left(1-t_{1}\right)\right], \\
& F_{g}\left(t_{2}\right)=\frac{1}{2}\left[F_{g}\left(t_{2}\right)+F_{g}\left(1-t_{2}\right)\right]
\end{aligned}
$$

From (2.14)-(2.16), we obtain that the function $F_{g}$ is decreasing on $\left[0, \frac{1}{2}\right]$. Since the function $F_{g}$ is symmetric about $\frac{1}{2}$ and the function $F_{g}$ is decreasing on $\left[0, \frac{1}{2}\right]$, we obtain that the function $F_{g}$ is increasing on $\left[\frac{1}{2}, 1\right]$. Using the symmetry and monotonicity of $F_{g}$, we derive the inequalities (2.10) and (2.11).

(3) Using the substitution rules for integration, we have the identity

$$
\begin{aligned}
F_{g}(t)= & \frac{1}{(d-c)^{2}} \int_{c}^{d} \int_{c}^{\frac{c+d}{2}}[f(\operatorname{tg}(s)+(1-t) g(u)) \\
& +f(\operatorname{tg}(s)+(1-t) g(c+d-u))] d u d s
\end{aligned}
$$

for all $t \in[0,1]$. Let $t \in[0,1]$. Since $g(u)+g(c+d-u)=2 g\left(\frac{c+d}{2}\right)(u \in[c, d])$, we obtain

$$
\begin{aligned}
& 2\left[\operatorname{tg}(s)+(1-t) g\left(\frac{c+d}{2}\right)\right] \\
& \quad=[\operatorname{tg}(s)+(1-t) g(u)]+[\operatorname{tg}(s)+(1-t) g(c+d-u)]
\end{aligned}
$$

and

$$
\begin{array}{r}
\left|\left[\operatorname{tg}(s)+(1-t) g\left(\frac{c+d}{2}\right)\right]-\left[\operatorname{tg}(s)+(1-t) g\left(\frac{c+d}{2}\right)\right]\right| \\
\quad \leq|[\operatorname{tg}(s)+(1-t) g(u)]-[\operatorname{tg}(s)+(1-t) g(c+d-u)]|
\end{array}
$$

for all $s \in[c, d]$ and $u \in\left[c, \frac{c+d}{2}\right]$. Therefore, by Lemma 2, the following inequality holds for all $s \in[c, d]$ and $u \in\left[c, \frac{c+d}{2}\right]$ :

$$
\begin{aligned}
& 2 f\left(\operatorname{tg}(s)+(1-t) g\left(\frac{c+d}{2}\right)\right) \\
& \quad \leq f(\operatorname{tg}(s)+(1-t) g(u))+f(\operatorname{tg}(s)+(1-t) g(c+d-u)),
\end{aligned}
$$

where $A=\operatorname{tg}(s)+(1-t) g(u), B=\operatorname{tg}(s)+(1-t) g(c+d-u)$ and $C=D=\operatorname{tg}(s)+(1-t) g\left(\frac{c+d}{2}\right)$. Dividing the above inequality by $(d-c)^{2}$, integrating it over $s$ on $[c, d]$, over $u$ on $\left[c, \frac{c+d}{2}\right]$ and using the above identity, we derive the inequality (2.12).

From the inequalities (2.6), (2.12) and the monotonicity of $H_{g}$, we derive the inequality (2.13).

This completes the proof.

Remark 5 In Theorem 4 , let $c=a, d=b$ and the function $g(s)=s$ on $[a, b]$. Then the functions $F_{g}(t)=F(t)(t \in[0,1])$ and Theorem 4 reduces to Theorem B. 


\section{Fejér type inequalities for general weights}

In this section, we establish some Fejér type inequalities for general weights which generalize Theorems D-F.

Theorem 5 Let the functions $f, g, p_{1}$ be defined as in the first page. Then:

(1) We have

$$
f\left(g\left(\frac{c+d}{2}\right)\right) \int_{c}^{d} p_{1}(s) d s \leq \int_{c}^{d} f(g(s)) p_{1}(s) d s .
$$

(2) As the function $g$ is monotonic on $[c, d]$, we obtain

$$
\int_{c}^{d} f(g(s)) p_{1}(s) d s \leq \frac{f(g(c))+f(g(d))}{2} \int_{c}^{d} p_{1}(s) d s
$$

Proof

(1) Using simple techniques of integration and the hypothesis of $p_{1}$, we have the identities

$$
\int_{c}^{d} f(g(s)) p_{1}(s) d s=\int_{c}^{\frac{c+d}{2}}[f(g(s))+f(g(c+d-s))] p_{1}(s) d s
$$

and

$$
\int_{c}^{\frac{c+d}{2}} p_{1}(s) d s=\frac{1}{2} \int_{c}^{d} p_{1}(s) d s
$$

Proceeding as in the proof of Theorem 1, we also obtain the inequality (2.4). Multiplying the inequality (2.4) by $p_{1}(s)$, integrating it over $s$ on $\left[c, \frac{c+d}{2}\right]$ and using the above identities, we obtain the inequality (3.1).

(2) Proceeding as in the proof of Theorem 1, we also obtain the inequality (2.5). Multiplying the inequality $(2.5)$ by $p_{1}(s)$, integrating it over $s$ on $\left[c, \frac{c+d}{2}\right]$ and using the above identities, we obtain the inequality (3.2). This completes the proof.

\section{Remark 6}

(1) Let $c=a, d=b$ and let the functions $g(s)=s$ and $p_{1}(s)=p(s)$ on $[a, b]$. Then Theorem 5 reduces to Fejér inequality (1.5).

(2) Let the function $p_{1}(s) \equiv \frac{1}{d-c}$ on $[c, d]$. Then Theorem 5 reduces to Theorem 1 .

Theorem 6 Let the functions $f, g, p_{1}, W H_{g}$ be defined as in the first page. Then:

(1) The function $W_{g}$ is convex on $[0,1]$.

(2) The function $W H_{g}$ is increasing on $[0,1]$ and, for all $t \in[0,1]$, we have

$$
\begin{aligned}
f\left(g\left(\frac{c+d}{2}\right)\right) \int_{c}^{d} p_{1}(s) d s & =W H_{g}(0) \\
& \leq W H_{g}(t) \\
& \leq W H_{g}(1)=\int_{c}^{d} f(g(s)) p_{1}(s) d s .
\end{aligned}
$$


(1) It is easily observed from the convexity of $f$ and the hypothesis of $p_{1}$ that the function $W H_{g}$ is convex on $[0,1]$.

(2) Using simple techniques of integration and the hypothesis of $p_{1}$, we have the following identity:

$$
\begin{aligned}
W H_{g}(t)= & \int_{c}^{\frac{c+d}{2}}\left[f\left(\operatorname{tg}(s)+(1-t) g\left(\frac{c+d}{2}\right)\right)\right. \\
& \left.+f\left(\operatorname{tg}(c+d-s)+(1-t) g\left(\frac{c+d}{2}\right)\right)\right] p_{1}(s) d s
\end{aligned}
$$

for all $t \in[0,1]$.

Let $t_{1}<t_{2}$ in $[0,1]$. Proceeding as in the proof of Theorem 2 , we also obtain the inequality (2.7). Multiplying the inequality (2.7) by $p_{1}(s)$, integrating it over $s$ on $\left[c, \frac{c+d}{2}\right]$ and using the above identity, we obtain

$$
W H_{g}\left(t_{1}\right) \leq W H_{g}\left(t_{2}\right) \text {. }
$$

Thus, the function $W H_{g}$ is increasing on $[0,1]$ and from which the inequality (3.5) holds. This completes the proof.

\section{Remark 7}

(1) In Theorem 6, the inequality (3.5) refines the inequality (3.1).

(2) Let the function $p_{1}(s) \equiv \frac{1}{d-c}$ on $[c, d]$. Then Theorem 6 reduces to Theorem 2.

Theorem 7 Let the functions $f, g, p_{1}, W P_{g}$ be defined as in the first and second pages. Then:

(1) The function $W P_{g}$ is convex on $[0,1]$.

(2) The function $W P_{g}$ is increasing on $[0,1]$ and, for all $t \in[0,1]$, we have

$$
\begin{aligned}
\int_{c}^{d} f(g(s)) p_{1}(s) d s & =W P_{g}(0) \\
& \leq W P_{g}(t) \\
& \leq W P_{g}(1)=\frac{f(g(c))+f(g(d))}{2} \int_{c}^{d} p_{1}(s) d s
\end{aligned}
$$

as the function $g$ is monotonic on $[c, d]$.

Proof

(1) It is easily observed from the convexity of $f$ and the hypothesis of $p_{1}$ that the function $W P_{g}$ is convex on $[0,1]$.

(2) Using simple techniques of integration and the hypothesis of $p_{1}$, we have the following identity:

$$
\begin{aligned}
W P_{g}(t)= & \int_{c}^{\frac{c+d}{2}}[f(\operatorname{tg}(c)+(1-t) g(s)) \\
& +f(\operatorname{tg}(d)+(1-t) g(c+d-s))] p_{1}(s) d s
\end{aligned}
$$

for all $t \in[0,1]$. 
Let $t_{1}<t_{2}$ in $[0,1]$. Proceeding as in the proof of Theorem 3 , we also obtain the inequality (2.9). Multiplying the inequality (2.9) by $p_{1}(s)$, integrating it over $s$ on $\left[c, \frac{c+d}{2}\right]$ and using the above identity, we obtain

$$
W P_{g}\left(t_{1}\right) \leq W P_{g}\left(t_{2}\right)
$$

Thus, the function $W P_{g}$ is increasing on $[0,1]$ and from which the inequality (3.6) holds. This completes the proof.

\section{Remark 8}

(1) In Theorem 7, the inequality (3.6) refines the inequality (3.2).

(2) Let the function $p_{1}(s) \equiv \frac{1}{d-c}$ on $[c, d]$. Then Theorem 7 reduces to Theorem 3.

Remark 9 Let $c=a, d=b$ and let the functions $g(s)=s$ and $p_{1}(s)=p(s)$ on $[a, b]$. Then Theorems 6 and 7 reduce to Theorem E.

Theorem 8 Let the functions $f, g, p_{1}, W H_{g}, W F_{g}$ be defined as in the first page. Then we have the following results:

(1) The function $W F_{g}$ is convex on $[0,1]$ and symmetric about $\frac{1}{2}$.

(2) The function $W F_{g}$ is decreasing on $\left[0, \frac{1}{2}\right]$ and increasing on $\left[\frac{1}{2}, 1\right]$,

$$
\sup _{t \in[0,1]} W F_{g}(t)=W F_{g}(0)=W F_{g}(1)=\int_{c}^{d} f(g(s)) p_{1}(s) d s
$$

and

$$
\inf _{t \in[0,1]} W_{g}(t)=W F_{g}\left(\frac{1}{2}\right)=\int_{c}^{d} \int_{c}^{d} f\left(\frac{g(s)+g(u)}{2}\right) p_{1}(s) p_{1}(u) d s d u
$$

(3) We have

$$
W H_{g}(t) \int_{c}^{d} p_{1}(s) d s \leq W F_{g}(t) \quad(t \in[0,1])
$$

and

$$
f\left(g\left(\frac{c+d}{2}\right)\right)\left(\int_{c}^{d} p_{1}(s) d s\right)^{2} \leq W F_{g}\left(\frac{1}{2}\right) .
$$

Proof

(1)-(2) Proceeding as in the proof of Theorem 4, the parts (1) and (2) hold.

(3) Using the substitution rules for integration and the hypothesis of $p_{1}$, we have the identity

$$
\begin{aligned}
W F_{g}(t)= & \int_{c}^{d} \int_{c}^{\frac{c+d}{2}}[f(\operatorname{tg}(s)+(1-t) g(u)) \\
& +f(\operatorname{tg}(s)+(1-t)(c+d-u))] p_{1}(u) p_{1}(s) d u d s
\end{aligned}
$$


for all $t \in[0,1]$. Proceeding as in the proof of Theorem 4 , we also obtain the inequality (2.17). Multiplying the inequality (2.17) by $p_{1}(u) p_{1}(s)$, integrating it over $s$ on $[c, d]$, over $u$ on $\left[c, \frac{c+d}{2}\right]$ and using the identities (3.4) and (3.9), we obtain the inequality (3.7).

From the inequalities (3.5), (3.7) and the monotonicity of $W_{g}$, we derive the inequality (3.8).

This completes the proof.

\section{Remark 10}

(1) Theorem 8 refines the inequality (3.1).

(2) Let the function $p_{1}(s) \equiv \frac{1}{d-c}$ on $[c, d]$. Then Theorem 8 reduces to Theorem 2 .

(3) Let $c=a, d=b$ and the functions $g(s)=s$ and $p_{1}(s)=p(s)$ on $[a, b]$. Then Theorem 8 reduces to Theorem $\mathrm{F}$.

\section{Competing interests}

The authors declare that they have no competing interests.

\section{Authors' contributions}

The authors did not provide this information.

\section{Author details}

${ }^{1}$ China University of Science and Technology, Nankang, Taipei, 11522, Taiwan. ²Department of Applied Mathematics, Aletheia University, Tamsui, New Taipei, 25103, Taiwan.

\section{Acknowledgements}

Dedicated to Professor Hari M Srivastava.

This research was partially supported by Grant NSC 101-2115-M-156-002.

Received: 23 December 2012 Accepted: 27 March 2013 Published: 15 April 2013

\section{References}

1. Hadamard, J: Étude sur les propriétés des fonctions entières en particulier d'une fonction considérée par Riemann. J. Math. Pures Appl. 58, 171-215 (1893)

2. Dragomir, SS: Two mappings in connection to Hadamard's inequalities. J. Math. Anal. Appl. 167, 49-56 (1992)

3. Dragomir, SS: A refinement of Hadamard's inequality for isotonic linear functionals. Tamkang. J. Math. 24, 101-106 (1993)

4. Dragomir, SS: On the Hadamard's inequality for convex on the co-ordinates in a rectangle from the plane. Taiwan. J. Math. 5(4), 775-788 (2001)

5. Dragomir, SS: Further properties of some mapping associated with Hermite-Hadamard inequalities. Tamkang. J. Math. 34(1), 45-57 (2003)

6. Dragomir, SS, Cho, Y-J, Kim, S-S: Inequalities of Hadamard's type for Lipschitzian mappings and their applications. J. Math. Anal. Appl. 245, 489-501 (2000)

7. Dragomir, SS, Milošević, DS, Sándor, J: On some refinements of Hadamard's inequalities and applications. Univ. Beograd. Publ. Elektrotehn. Fak. Ser. Mat. 4, 3-10 (1993)

8. Fejér, L: Über die Fourierreihen, II. Math. Naturwiss Anz Ungar. Akad. Wiss. 24, 369-390 (1906) (In Hungarian)

9. Hwang, D-Y, Tseng, K-L, Yang, G-S: Some Hadamard's inequalities for co-ordinated convex functions in a rectangle from the plane. Taiwan. J. Math. 11(1), 63-73 (2007)

10. Tseng, K-L, Hwang, S-R, Dragomir, SS: On some new inequalities of Hermite-Hadamard-Fejér type involving convex functions. Demonstr. Math. XL(1), 51-64 (2007)

11. Tseng, K-L, Yang, G-S, Hsu, K-C: On some inequalities of Hadamard's type and applications. Taiwan. J. Math. 13(6B), 1929-1948 (2009)

12. Yang, G-S, Hong, M-C: A note on Hadamard's inequality. Tamkang. J. Math. 28(1), 33-37 (1997)

13. Yang, G-S, Tseng, K-L: On certain integral inequalities related to Hermite-Hadamard inequalities. J. Math. Anal. Appl. 239, 180-187 (1999)

14. Yang, G-S, Tseng, K-L: Inequalities of Hadamard's type for Lipschitzian mappings. J. Math. Anal. Appl. 260, 230-238 (2001)

15. Yang, G-S, Tseng, K-L: On certain multiple integral inequalities related to Hermite-Hadamard inequalities. Util. Math. 62, 131-142 (2002)

16. Yang, G-S, Tseng, K-L: Inequalities of Hermite-Hadamard-Fejér type for convex functions and Lipschitzian functions. Taiwan. J. Math. 7(3), 433-440 (2003) 